\title{
Structural damage detection using ARMAX time series models and cepstral distances
}

\author{
$\mathrm{K}_{\text {LAKSHMI }}{ }^{\left.1,2, *^{(}\right)}$and A RAMA MOHAN RAO ${ }^{1,2}$ \\ ${ }^{1}$ Academy of Scientific and Innovative Research, Chennai 600 113, India \\ ${ }^{2}$ CSIR-Structural Engineering Research Centre, Chennai 600113, India \\ e-mail: lakshmik@serc.res.in; arm@serc.res.in
}

MS received 11 June 2015; revised 30 November 2015; accepted 2 May 2016

\begin{abstract}
A novel damage detection algorithm for structural health monitoring using time series model is presented. The proposed algorithm uses output-only acceleration time series obtained from sensors on the structure which are fitted using Auto-regressive moving-average with exogenous inputs (ARMAX) model. The algorithm uses Cepstral distances between the ARMAX models of decorrelated data obtained from healthy and any other current condition of the structure as the damage indicator. A numerical model of a simply supported beam with variations due to temperature and operating conditions along with measurement noise is used to demonstrate the effectiveness of the proposed damage diagnostic technique using the ARMAX time series models and their Cepstral distances with novelty indices. The effectiveness of the proposed method is validated using the benchmark data of the 8-DOF system made available to public by the Engineering Institute of LANL and the simulated vibration data obtained from the FEM model of IASC-ASCE 12-DOF steel frame. The results of the studies indicate that the proposed algorithm is robust in identifying the damage from the acceleration data contaminated with noise under varied environmental and operational conditions.
\end{abstract}

Keywords. Structural health monitoring; damage detection; time series models; ARMAX; 8-DOF benchmark data; IASC-ASCE steel frame.

\section{Introduction}

With the increasing necessity of automated structural health monitoring systems, wireless sensing unit constructed with advanced embedded system technologies is developing at a much faster pace. On the other hand, Structural health monitoring (SHM) is gaining advantage of these technologies in sensors, wireless networks, and data analysis methods to monitor the civil infrastructures and increase the life safety of them. Recent research has demonstrated that wireless sensing networks can be successfully used for structural health monitoring [1,2]. As a part of SHM, the goal of damage diagnosis is to detect, localize, and quantify structural damage due to corrosion, earthquakes, etc.

One method of detecting damage in a structure is by measuring the structure's vibration characteristics through strain or acceleration. The premise is that the changes in structural properties caused by damage will change the way a structure responds to ambient motions. Most of the currently available damage detection methods fall under the classification of global methods, where the dynamic properties (natural frequencies and mode shapes) are obtained for the entire structure from the input-output data using a

*For correspondence global structural analysis [3]. The global damage measures are not only insensitive to minor damage and local damage but also involve finite element modeling and system identification methods, which can be computationally expensive. Recently, statistical signal processing techniques, which rely on the signatures obtained from the recorded vibration, strain or other data to extract features that change with the onset of damage are becoming popular [4]. These methods are found to avoid the complexity of global system identification techniques and serve as a technique for data reduction that will minimize the amount of data needed to be transmitted by the radio of the wireless monitoring node. However, there remains a trade-off between the requirement of a more sophisticated monitoring hardware to be deployed on the system and a sophisticated data analysis procedure that can be used to interrogate the measured data.

Parametric time series methods for SHM are those based on corresponding time series representations, such as the autoregressive models [5] and autoregressive moving average (ARMA) representation [6,7]. These types of methods have attracted considerable attention and their principles have been used in a number of studies. Sohn et al [4] used a combination of AR models and AR models with exogenous inputs to normalize the data collected from a patrol boat under unknown operational and environmental 
conditions, and they separated the data from damage conditions from that of undamaged conditions in the context of a statistical pattern recognition problem. Carden and Brownjohn [8] classified different states of several structures using autoregressive moving average (ARMA) models. They mentioned that vector ARMA (ARMAV) models can provide spatial information about the structural vibration in addition to the natural frequencies. Zhang [9] used an approach based on AR-ARX models to identify the damage location in a steel beam. Bodeux and Golinval [10] used ARMAV models for damage detection in the 'SteelQuake' structure in the framework of a European benchmark test structure. A two-stage damage diagnosis strategy is proposed by Zheng and Mita [11]. Damage existence is determined in the first stage using a damage indicator defined as the distance between two ARMA models, while, in a second stage, damage localization is achieved via prewhitening filters. Sakellariou and Fassois [12] employed Output Error (OE) models and statistical hypothesis testing procedures utilizing the corresponding model parameter vectors, in order to achieve damage diagnosis in structures under earthquake excitation. Fung et al [13] developed and implemented ARMAX and NARMAX models for the onestep ahead forecasting of both the radial and longitudinal errors of an experimental lathe turning machine. The parameters of the ARMAX and NARMAX structures were estimated by the recursive extended least square (RELS) method and the neural network (NN) method, respectively. They concluded that using the nonlinear NARMAX representation and the $\mathrm{NN}$ identification method, future machining errors can be accurately forecasted. Zhenhua et al [14] used a substructure algorithm to divide a complete structure into substructures. For each substructure, an ARMAX model is used to extract the modal information. The extent of damage is measured by using the squared original frequency and the squared damaged frequency. In this paper, a novel damage detection algorithm for structural health monitoring using time series model is presented. The proposed algorithm uses output-only acceleration time series obtained from sensors on the structure which are fitted using Auto-regressive movingaverage with exogenous inputs (ARMAX) model, after decorrelation process. The algorithm then uses Cepstral distances of two ARMAX models obtained from a healthy and an unknown current condition of the structure, as the damage indicator. The robustness of the algorithm in differentiating damage from variability that arise due to environment, measurement, operation, etc., is enhanced by employing the control limits using novelty indices. A numerical model of a simply supported beam with variations due to temperature and operating conditions along with measurement noise is used to demonstrate the effectiveness of the proposed damage diagnostic technique using the ARMAX time series models and their Cepstral distances with novelty indices. The effectiveness of the proposed method is validated using the benchmark data of the
8-DOF system made available to public by the Engineering Institute of LANL and the simulated vibration data obtained from the 12-DOF FEM model of IASC-ASCE steel frame. The results of the studies indicate that the proposed algorithm is robust in identifying the damage from the acceleration data contaminated with noise under varied environmental and operational conditions.

\section{Time series analysis}

A time series is a collection of quantitative observations that are evenly spaced in time and measured successively. Time series are analyzed in order to understand the underlying structure and function that produce the observations. Understanding the mechanisms of a time series allows a mathematical model to be developed that explains the data in such a way that prediction, monitoring, or control can occur. Observations made over time can be either discrete or continuous. Both types of observations can be equally spaced, unequally spaced, or have missing data. Discrete measurements can be recorded at any time interval, but are most often taken at evenly spaced intervals. Continuous measurements can be spaced randomly in time, such as measuring earthquakes as they occur because an instrument is constantly recording, or can entail constant measurement of a natural phenomenon such as air temperature, or a process such as the velocity of an airplane. Time series are very complex because each observation, to some extent, is dependent upon the previous observation, and often is influenced by more than one previous observation. The challenge of time series analysis is to model the underlying mechanisms.

\subsection{Time series analysis in SHM}

SHM methods based on time series, exclusively use the output-only vibration time history data which are continuous and stochastic, collected from various sensors on the structure of interest and represent it as outputs of a selected time series model. Time series models rely on the fact that the value of the measured response of the linear system at any time can be predicted based on a linear combination of its values at a previous time and some random errors. The analogy behind using time series models for vibrationbased damage identification of structures is that if a time series model is fitted to the vibration responses of the structure, the obtained coefficients and properties of the model can capture the dynamic characteristics of the structure. Then, any deviation from the obtained model can be a sign of change or damage in the structure. Time series analysis in SHM offers unique advantages [6] such as

- No need for physics based or analytical, such as finite element (FE) models.

- No need for complete structural models. 
- Inherent accounting for uncertainties (measurement, environmental, operational and so on) through statistical tools.

- Statistical decision making with specified performance characteristics.

- Effective operation even in the "low" frequency range.

- Effective use of naturally obtained random vibration signals without interrupting the normal operation of the structure.

Inspired by the ease and advantages of the technique, time series based SHM is being employed in numerous applications by deriving meaningful damage features extracted from appropriately selected time series model. In the search for more sensitive damage features that can capture more information from the measured vibration time history responses of structures, researchers propose to use different types of time series models. Many existing SHM feature extraction methodologies are based on fitting linear models (i.e. a modal model) to the measured system response data before and after damage. Changes in the parameters of these models are then used as indicators of damage. This physics-based modeling approach has been extended to data-based time-series models, where, in addition to model parameters, residual errors between measured and predicted responses are used as damage-sensitive features. There are three basic types of linear models: autoregressive (AR), moving-average (MA), and ARMA models. These models provide a good first-order approximations to the dynamics of the data-generating process. Among the various statistical models that are reviewed in time series analysis, the simplest one is the auto-regressive (AR) model, with an output linearly dependent on preceding values. When the system input is available, a nonlinear auto-regressive with exogenous input (ARX) model can be constructed.

2.1a AR model: The AR model structure is a process model used in the generation of models where outputs are only dependent on previous outputs. The AR models are the most popular time-series models, as they can be fully estimated and tested within the framework of least-squares regression. A series $X_{t}$ is said to follow an AR model if

$$
X_{t}=\sum_{j=1}^{p} \phi_{t j} X_{t-j}+e_{t}
$$

where $p$ is the AR order, $\phi_{t j}$ are the AR coefficients and $e_{t}$ is the prediction error.

This is $\mathrm{AR}(\mathrm{p})$ model or the autoregressive model of order $p$. The error $e_{t}$ is usually specified as white noise, i.e. as uncorrelated over time with a constant variance and mean zero. Sometimes, time independence is also required. It is convenient to write the model in lag operators, defined as $B X_{t}=X_{t-1}$ as

$$
\begin{aligned}
& X_{t}=\varphi_{1} B_{1} X_{t}+\varphi_{2} B_{2} X_{t}+\cdots+\varphi_{p} B_{p} X_{t}+e_{t} \\
& \quad\left(1-\varphi_{1} B_{1}-\varphi_{2} B_{2}-\cdots-\varphi_{p} B_{p}\right) X_{t}=e_{t} \\
& \quad \varphi(B) X_{t}=e_{t} .
\end{aligned}
$$

2.1b ARX model: The popular ARX model is the simplest model incorporating the stimulus/input signal. In general ARX model is represented as follows.

$$
x_{t}=\sum_{i=1}^{n a} \alpha_{i} x_{t-i}+\sum_{j=1}^{n b} \beta_{j} u_{t-j-n k}+\varepsilon_{t}
$$

which relates the current output $x_{t}$ to a finite number of past outputs $x_{t-i}$ and inputs $u_{t-j}$. The structure is thus entirely defined by the three integers $n a, n b$, and $n k$. $n a$ is equal to the number of poles and $n b-1$ is the number of zeroes, while $n k$ is the pure time-delay (the dead-time) in the system. The one-step prediction error $\varepsilon_{t}$ of an ARX model over a dataset is generally referred to as model residuals. The model parameters are estimated by methods based on least squares, exact likelihood and Yule-Walker equation estimates.

ARX model is popularly employed in many of the damage detection methods in SHM due to the fact that it is simple like AR model and requires the signal from only one sensor thereby reducing the computation cost. The ARX model is the simplest model incorporating a stimulus signal without taking disturbances into account. The estimation of the ARX model is the most efficient of the polynomial estimation methods because it provides a unique solution by solving the linear regression equations in an analytic form, satisfying the global minimum of the loss function. On the other hand, unlike in ARX model, the transfer function of the deterministic part of the system and the transfer function of the stochastic part of the system do not have the same set of poles, unless there exists a good constant signal-to-noise ratio. For practical measurements, where the data is collected from the field/experiment, maintaining constant SNR is quite infeasible.

Therefore, a suitable alteration to this model is to include the disturbances created from the dynamic system and this results in the auto-regressive moving average with exogenous input (ARMAX) model. An ARMAX model can be seen as an effective model, where all aspects of the AR, ARX and ARMA models are included. ARMAX creates a generalized mathematical description of the nonlinear dynamical system with stochastic noise and integrates the variation of input parameters into the system model [15]. The main advantage of ARMAX model is that, it inherently mitigates for signals with noise from various sources, providing unbiased parameter estimates.

In this paper, a damage detection technique based on Cepstral distances between ARMAX models is presented. Novelty index is used to calculate the control limits to differentiate the damage from the effects of variability like 
environmental, operational and measurement noise. The robustness of the technique to handle measurement noise, environmental and/or operational variability is verified using the acceleration data from the numerically simulated as well as the standard benchmark problems.

\section{Proposed method}

The proposed damage localization methodology, based on the Cepstral distance between ARMAX time series models, obtained from the data corresponding to different states of the structure, is described in this section. Initially, healthy data is created from the acceleration signals obtained in known healthy state of the structure. Similarly, several databases which consist of the acceleration data from the unknown condition or otherwise called as the current condition of the structure are formed. By philosophy, these unknown signals are processed by comparing with the healthy data to arrive at a meaningful conclusion on the condition of the structure using a suitable ARMAX model. To compare two ARMAX models, a metric based on power cepstrum expressed in terms of the variance of the unknown excitation and the poles and zeroes of the model [16] are used. The power cepstrum of a signal is defined as the squared magnitude of the inverse Fourier transform of the logarithm of the squared magnitude of the Fourier transform of a signal. The Cepstral distance between the time series models is referred to as the damage indicator, which is representative of the damage location. The damage metric based on Cepstral distances requires models with mutually independent input processes with the same variance. Therefore, to minimize the correlation, decorrelation processing of the data is required before calculating the damage indicators. The stochastic excitations acting on the structures in real life, such as wind and traffic loadings are mutually dependent and correlated. Decorrelation technique is applied to decorrelate the sensor signals from vibration structure before conducting the proposed damage detection methodology.

\subsection{Decorrelation}

In the process of decorrelation, the m-dimensional sensor signals $x(t)$ are pre-processed by using the following transformation

$$
y(t)=M x(t)
$$

where $y(t)$ denotes the de-correlated signals, and $M$ is the $m \times m$ matrix. For this purpose, the matrix $M$ is chosen so that the covariance matrix $R_{y y}$ becomes the unit matrix $I$ as.

$$
R_{y y}=E\left\{y y^{T}\right\}=E\left\{M x x^{T} M^{T}\right\}=M R_{x x} M^{T}=I .
$$

Usually, the covariance matrix for sensor signals $x(t)$ is symmetric positive definite and it is therefore decomposed as follows:

$$
R_{x x}=\psi_{x} \omega_{x}^{1 / 2} \omega_{x}^{1 / 2} \psi_{x}^{T}
$$

where $\psi_{x}$ is an orthogonal matrix and, $\omega_{x}=\operatorname{diag}\left\{\lambda_{1}, \lambda_{2}, \cdots\right.$ $\left.\lambda_{\mathrm{m}}\right\}$ is a diagonal matrix with positive eigenvalues $\lambda_{1} \geq \lambda_{2}$ $\geq, \geq \lambda_{\mathrm{m}}>0$. Hence, under the condition that the covariance matrix is positive definite, the required decorrelation matrix can be computed as follows:

$$
M=V \omega_{x}^{-1 / 2} \psi_{x}^{T}
$$

where $V$ is an arbitrary orthogonal matrix. Thus the components of the de-correlated signals $y(t)$ in Eq. (4) are mutually uncorrelated and they have unit variance.

After decorrelation process, the data in all datasets are divided into smaller data samples. Segmenting the vibration responses is beneficial in representing different portions of the responses obtained under a varying load condition as well as including the effect of uncertainty in data prediction using time series models.

\subsection{Normalisation of current subset}

In the process of normalization, ARMAX models are fitted to all the segmented data samples from healthy and current datasets. Unlike the ARX model, the ARMAX model includes disturbance dynamics. An ARMAX process is given by

$$
A\left(q^{-1}\right) x[t]=B\left(q^{-1}\right) u\left[t-n_{t}\right]+C\left(q^{-1}\right) e_{x}[t]
$$

where $e_{x}[t]$ is the error between the measured signal and the output from the prediction model and $n_{t}$ is the time delay, which, in this case, is set to $1 . A\left(q^{-1}\right), B\left(q^{-1}\right)$, and $C\left(q^{-1}\right)$ are the polynomials in the delay operator $q^{-1}$.

The acceleration signal of adjacent channels in a healthy dataset is taken as the input series for all the ARMAX model construction of different samples. Varying environmental and operational (loading) conditions can significantly affect the vibration responses of structures. The normalization procedure is performed to match the data samples in different datasets that correspond to similar operational conditions and measurement noise. For this purpose, the number of data samples that are segmented in the healthy dataset, is much larger than the number of data samples in the current datasets. Therefore, the data samples in the healthy dataset represent the vibration response of the beam collected under a variety of loading conditions and measurement noise. For each data subset in current dataset, a data subset is matched from the healthy dataset. The AR coefficients of the ARMAX model are used to match any two data subsets on the basis of their correlation value. After normalisation, damage indices are calculated from the matched healthy and current data subset by using the cepstral distances of the two ARMAX models of those data subsets. 


\subsection{Damage indicator}

The system transfer function of ARMAX process has the form in the $z$ domain [17].

$$
H(Z)=\left[\begin{array}{ll}
A^{-1}(z) B(z) & A^{-1}(z) C(z)
\end{array}\right] .
$$

Considering the second part of the system transfer function where the AR and MA part of the system is available in terms of poles, $\alpha$ and zeros, $\beta$,

$$
H(z)=\frac{\sum_{i=0}^{n c} c(i) z^{-i}}{\sum_{i=0}^{n a} a(i) z^{-i}}=\frac{\prod_{i-1}^{n c}\left(1-\beta(i) z^{-i}\right)}{\prod_{i-1}^{n a}\left(1-\alpha(i) z^{-i}\right)} .
$$

For two ARMA models $M_{1}$ and $M_{2}$ with the associated cepstrum coefficients $c c_{1}(n)$ and $c c_{2}(n)$, the cepstral distance between them is defined by a weighted Euclidean distance between cepstrums [18].

$$
d\left(M_{1}, M_{2}\right)^{2}=\sum_{n=1}^{\infty} n\left|c c_{1}(n)-c c_{2}(n)\right|^{2}
$$

where $c c(n)$ are the cepstrum coefficients given by the following expression.

$$
c c(n)=\left\{\frac{1}{|n|}\left\{\sum_{i=1}^{p} \alpha^{|n|}(i)-\sum_{i=1}^{q} \beta^{|n|}(i)\right\}\right\}, \quad n \neq 0 .
$$

The distance measure obtained from Eq. (11) is used as damage localization index.

\subsection{Handling environmental variability}

The presence of environmental variability in addition to the operational variability is inevitable in the data collected from the civil structures. The presence of environmental variability alters the mechanical properties of the material. For example, the elasticity matrix of steel shown in figure 1

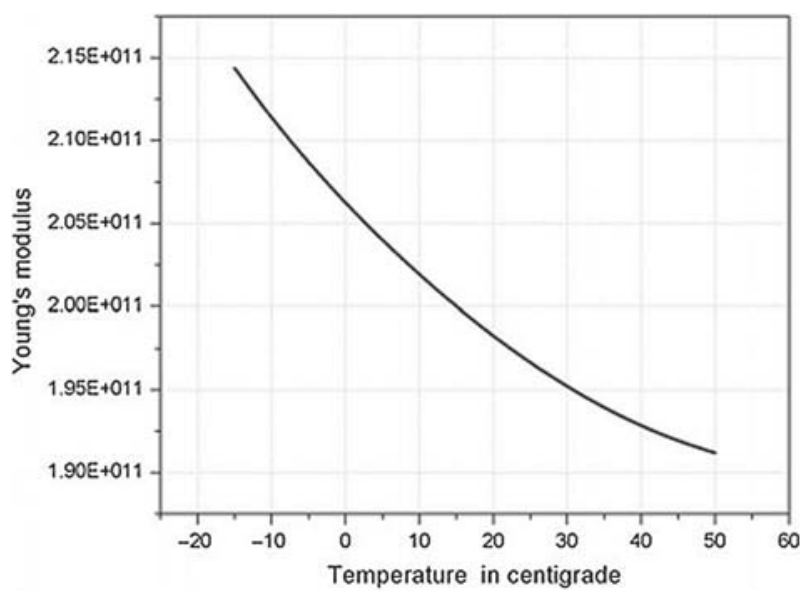

Figure 1. Variation of Young's modulus of steel with temperature [29]. clearly indicates that the Young's modulus of the material varies with the temperature. Therefore, it is evident that the change in the vibration characteristics of the structure may even be caused due to the temperature alterations. This may lead to false alarms during online monitoring. Generally, the operational variability and measurement noise are handled by normalization, as discussed in the section 3.2, using the process of matching. However, due to the presence of various external variability on a structure, it is difficult to match a new data subset, exactly with a subset in the healthy data pool to extract the damage features. Therefore, it becomes a close match instead of the exact match of data subsets. Consequently, the damage indices obtained from the datasets which are not exactly matched with each other, but only with a close match, give rise to a situation where the damage index obtained becomes inaccurate and leads to false alarms. Therefore, to alleviate the possibility of false indication and to handle the effects of variability due to environment, operation and measurement noise more effectively, a method based on the novelty index is proposed in this paper.

Novelty detection first builds an internal representation of the system's normal condition, and then examines subsequent data to see if they significantly depart from a normal condition. One major advantage of this novelty detection is that statistical model building for damage classification is based only on data from the undamaged system. However, the success or failure of the novelty detection is contingent on the accuracy of the description of the normal condition. In reality, the normal condition of the system may experience a wide range of variation due to operational and environmental changes of the system. Hence we establish control limits considering that the variations due to operational and environmental changes of the system are normally distributed. In this proposed method, the discordancy of a candidate outlier is measured by the Cepstral distance between the current data subset and the matched baseline data subset. Once the Cepstral distance is computed, it is checked against a threshold value. This threshold value is calculated by the novelty indices.

In this proposed method, the Cepstral distance, $D 1_{-} h$, between the ARMAX models of the first healthy subset and other subsets of the reference subset data are calculated using Eq. (11). Similarly, the distance measures of every healthy subset from all other subsets are calculated. Then novelty indices on these distance measures are constructed by establishing the control limits, considering that the damage indices are normally distributed.

An X-bar control chart is constructed by drawing the upper control limit (UCL) as

$$
\mathrm{UCL}=\operatorname{Mean}\left[d_{-} h\right]+3 \times\left(\text { Standard deviation }\left[d_{-} h\right]\right)
$$

where ' $d \_h$ ' denotes the vector of damage indices, calculated from the data subsets obtained when the structure is healthy, using Eq. (11). The presence of the damage index 

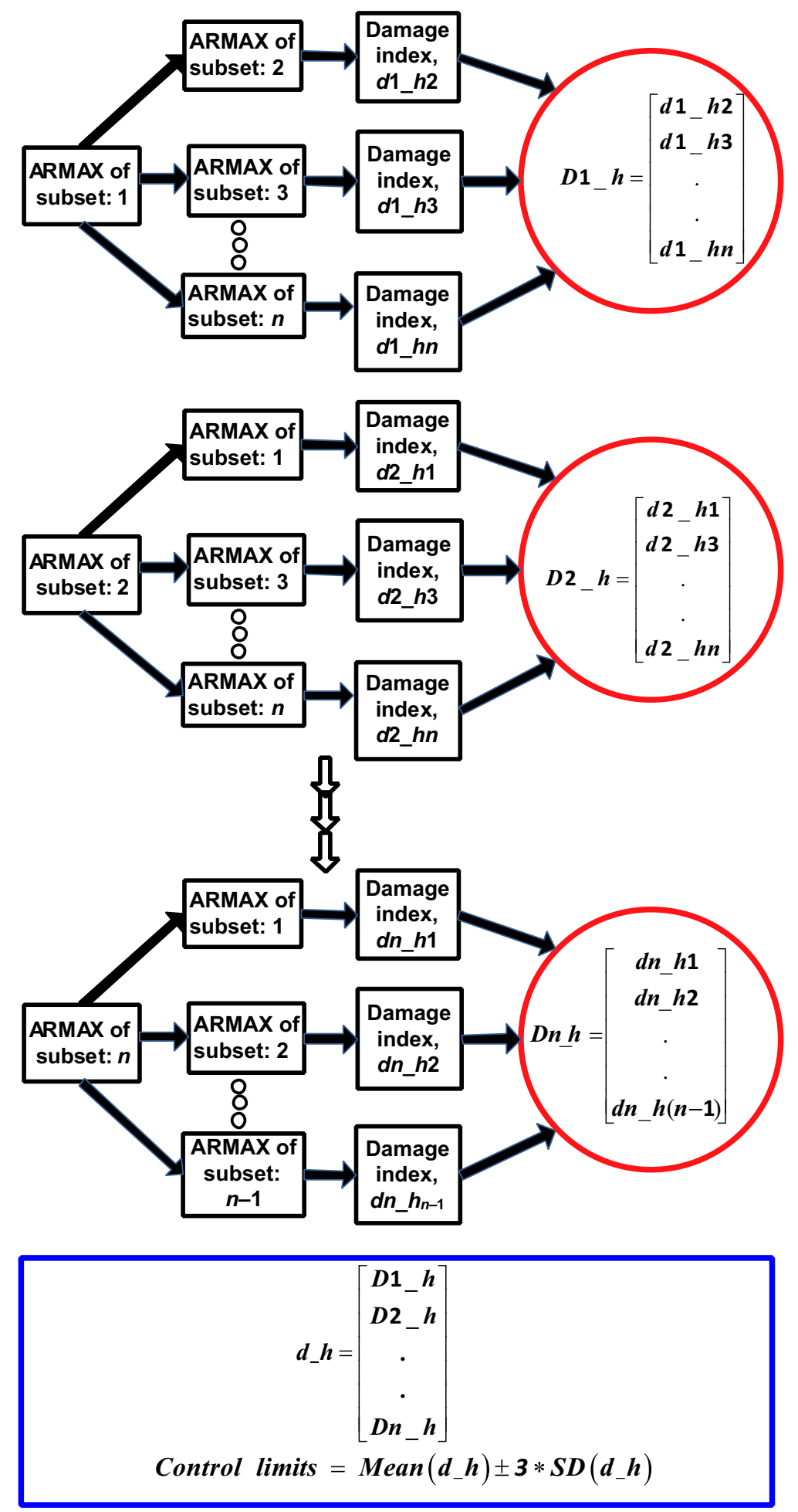

Figure 2. Control limits for novelty analysis using healthy subsets.

of a new current data subset, beyond the upper control limit is considered to be the direct reflection of the presence of damage in the structure of interest. For the benefit of readers, a schematic view of calculation of control limits is shown in figure 2. The flowchart of the proposed methodology is shown in figure 3 . 

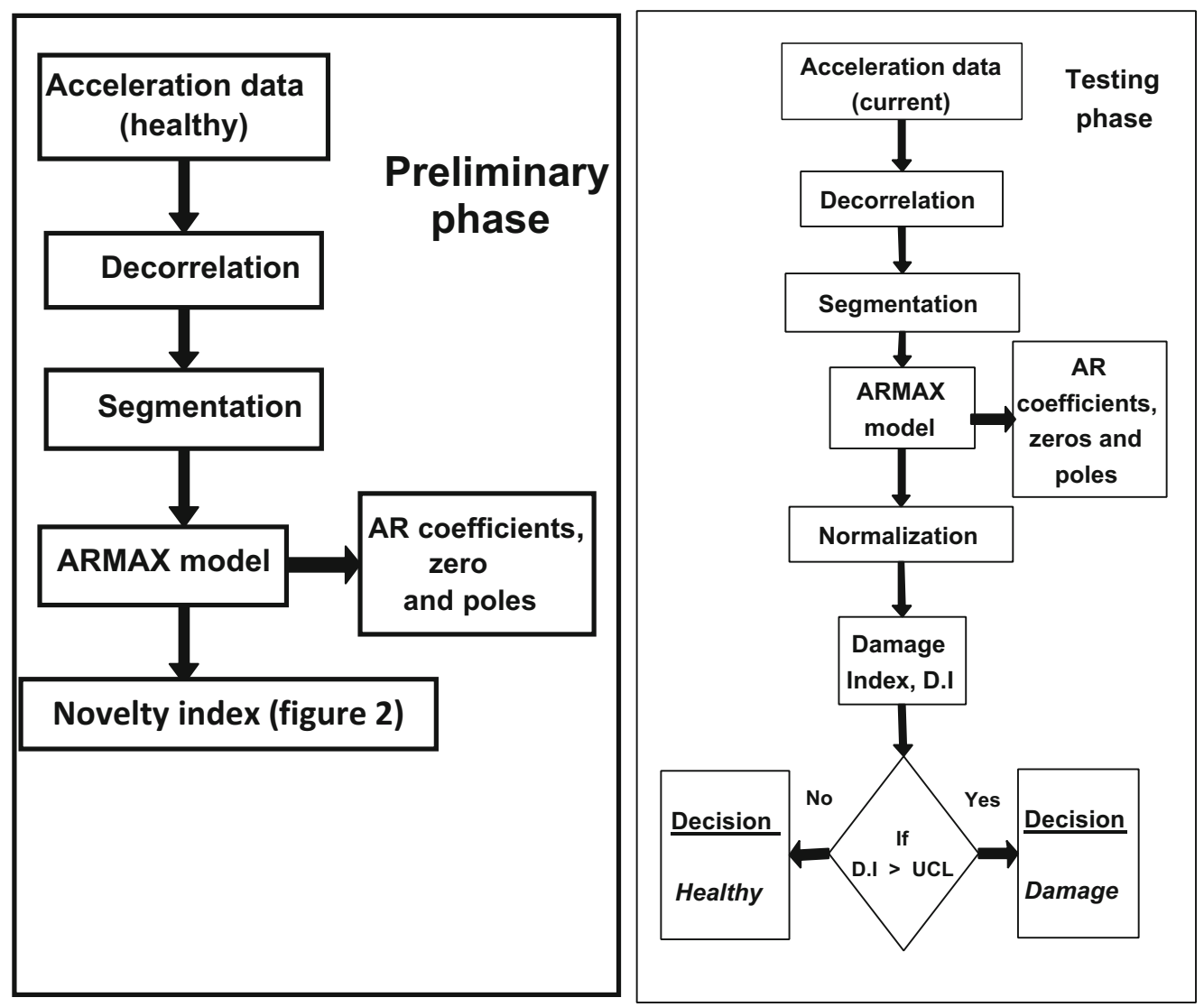

Figure 3. Damage detection methodology.

\section{Numerical studies}

A numerical model of a simply supported beam with variations due to temperature and operating conditions along with measurement noise is used to demonstrate the effectiveness of the proposed damage diagnostic technique using the ARMAX time series models and their Cepstral distances with novelty indices. The experimental data of 8-DOF benchmark problem provided by EI-LANL and the numerically simulated responses of a four-storey, IASCASCE SHM benchmark problem by Earthquake Engineering Research Laboratory of the University of British Columbia (UBC) are used further for the validation of the proposed technique to handle operational variability and noise.

\subsection{Simply supported beam}

A numerical model of a simply supported beam girder as shown in figure 4 with a span of $10.0 \mathrm{~m}$ is considered as the first example. For the purpose of numerical simulation studies, the beam is discretized into 20 elements, where each node except the support nodes carries a sensor. The total number of sensor locations works out to 19. The material and geometrical properties are also shown in figure 4 . The beam is excited using a random dynamic load which is stochastic in nature. The acceleration time-history response is computed using finite element analysis with Newmark's time-marching scheme. The sampling rate is chosen as $2000 \mathrm{~Hz}$. This sampling rate was enough, according to the Nyquist criterion to capture all the frequency content of the vibration responses in the range of 0-500 Hz.

4.1a Generation of healthy and current data: Baseline acceleration time-history data for healthy structure is generated with random load and with varied temperatures ranging from -15 to $50^{\circ} \mathrm{C}$. The simply supported beam is made up of steel and the elasticity matrix is assumed to be temperature dependent as illustrated in figure 1 . One of the main issues related to structural damage-diagnostic techniques, when applied to real situations, is their sensitivity to noise. In view of this, white Gaussian noise is added in the form of signal-to-noise ratio (SNR) to the acceleration time history before they are processed. SNR defines the amplitude of the noise with respect to that of the clean signal. Moreover, the noisy sequences affecting different nodes are uncorrelated, in this way severe experimental conditions are simulated. 


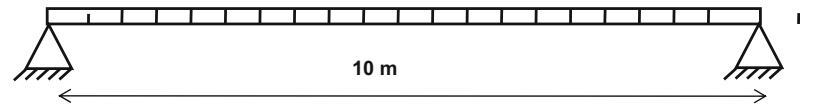

Elastic modulus $=205 \mathrm{GPa} ; \quad$ Cross sectional Area $=0.225 \mathrm{~m}^{2}$

Mass density $=7850 \mathrm{~kg} / \mathrm{m}^{3 ;}$ Moment of inertia $=0.000562 \mathrm{~m}^{4}$

Figure 4. Simply supported beam.

The generated data is partitioned into subsets of 500 time points. Each subset is added with varied noise levels (with SNR values ranging from 40 to 60 ) to exactly simulate the measurement noise and other uncertainties in measurement. In this numerical example, 500 such subsets are generated with varied operational conditions, noise and temperature ranges, to simulate an actual ambient environment. The damage indices are calculated by considering each healthy subset against the other subsets as discussed in section 3.4 using the Cepstral distance measures of those subsets. Therefore, for each healthy subset of a channel, the number of damage indices, equal to one less than the total healthy subsets, is generated. Similarly, the damage indices for all the healthy subsets of all channels are evaluated and the upper control limit is established from all these damage indices. The upper control limit is evaluated to be 3.237 for this numerical example.

Three current datasets of length $2 \mathrm{~s}$ are generated with SNR of 40, 50 and 60 respectively with varied loading and temperature. The generated current data is partitioned into 8 subsets of 500 sample points. During the generation of the current data, a hypothetical damage scenario is introduced in the beam by means of a reduction of stiffness by $20 \%$ in element no. 5, after $1 \mathrm{~s}$ (i.e. after the fourth subset). This forms the single damage scenario considered for the study. To simulate a multiple damage scenario, a current data with a stiffness reduction of $22 \%$, after $1 \mathrm{~s}$ of healthy data, at element 3 and 12 is generated. Typical raw acceleration data of a matched healthy (reference data) and current subset generated from the center node of simply supported beam is illustrated in figure 5 .

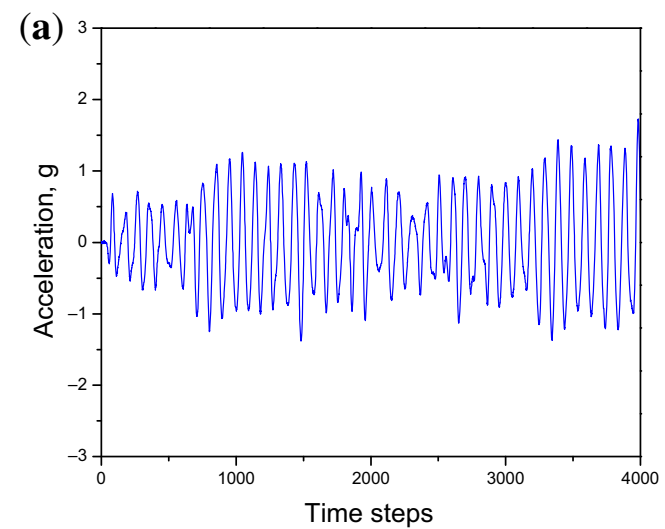

4.1b Damage diagnosis: Once the novelty indices are established, they are used further for damage detection using the current data subsets. The first current data with SNR 40 is subjected to the damage detection process. Every subset is fitted to the ARMAX model to obtain AR coefficients, zeroes and poles of the subset. Using the AR coefficients thus obtained, they are subjected to the normalization process described in section 3.2 to match appropriately with any of the healthy subsets. Using the matched healthy and current subset, the damage indices are obtained from the cepstral distance measure. The indices obtained for the first current data subset are shown in figure 6(a). It can be seen that the indices are within the UCL (indicated using a solid line) and, therefore, it can be concluded that the structure is intact. Similarly, the next three subsets showed the same trend where all the indices are found to be within the control limits. While processing the fifth subset, where the damage is actually introduced, the damage index at the 5th node shows relatively higher magnitude but do not indicate itself as an outlier (i.e. does not fall beyond the control limit). Further, the presence of other peaks in the figure demands a clear threshold to decide on the indication of damage. Unfortunately, in this example, the threshold obtained through novelty analysis also fails to indicate the damage location precisely. This is shown clearly in figure 6(b). The damage indices of next three subsets also are seen to follow the same trend. However, they are not shown here to concise the number of figures.

Similarly, the other two current data with SNR 50 and 60 , respectively, are used for damage detection process. The damage indices of the first and the fifth subset in each case are shown in figure 6(c)-6(f) respectively. From Figure 6, it can be seen that even though there is an increase in the magnitude of the damage indices at the location of damage, they are not falling beyond the control limit (UCL) as outliers. Also, the presence of more than one peak in the damage index plot does not indicate the exact location of damage. In addition to the above, it is clearly seen that the

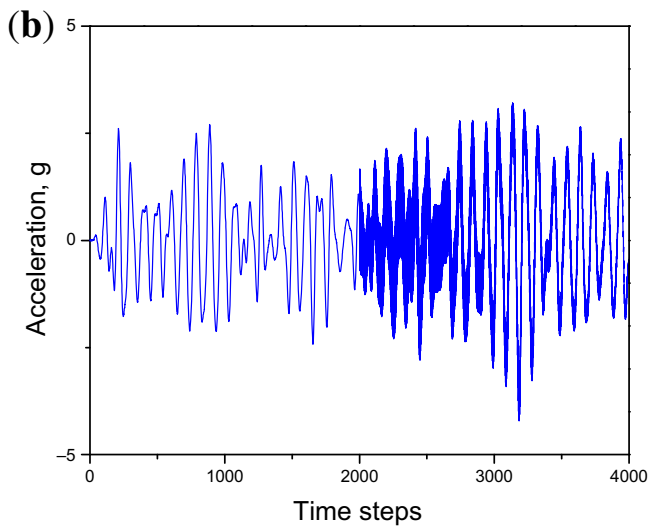

Figure 5. Typical raw accelerations of the center node of simply supported beam: (a) healthy data and (b) current data with SNR 40 . 
(a)

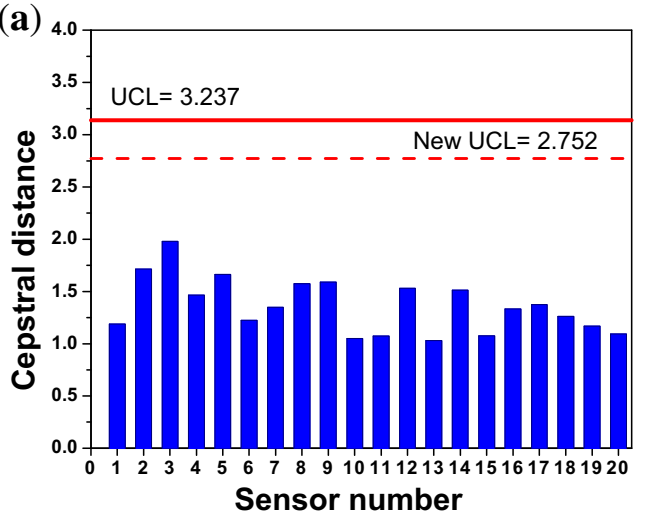

(c)

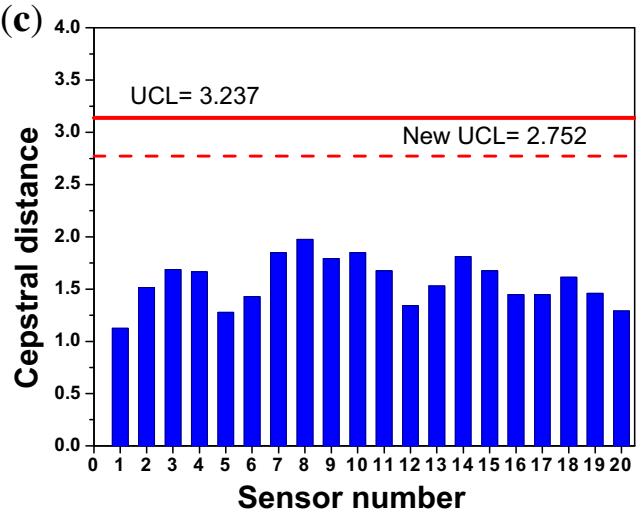

(e)

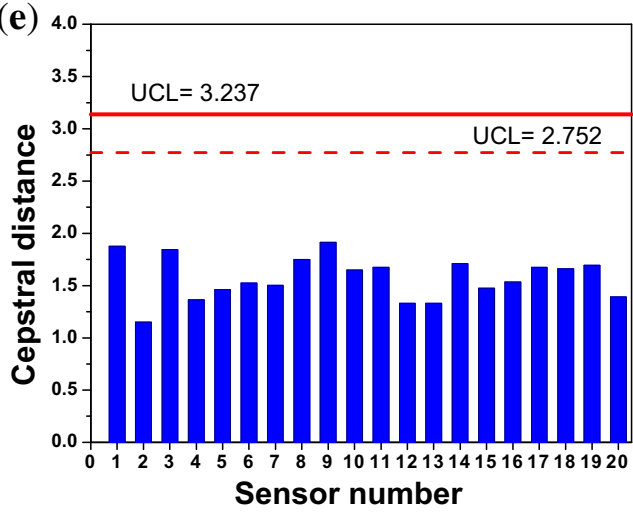

(b)

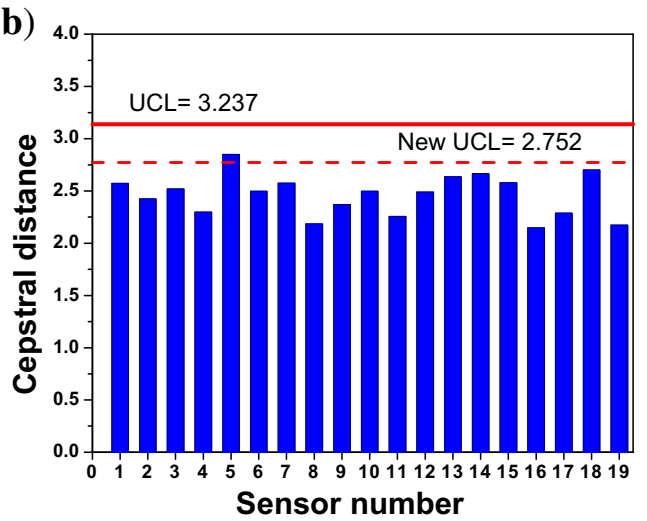

(d)

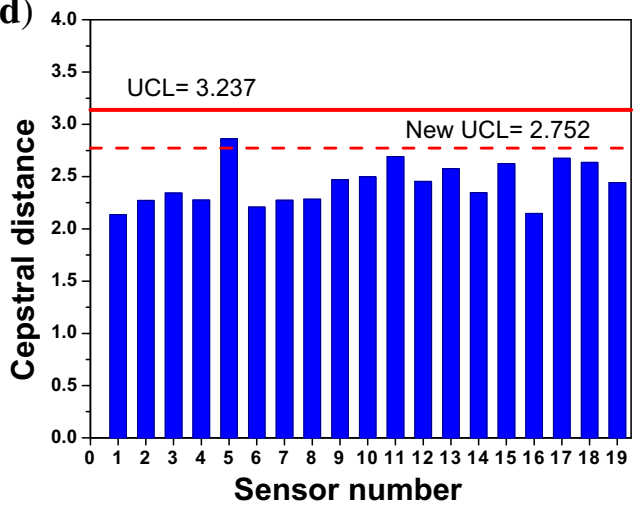

(f)

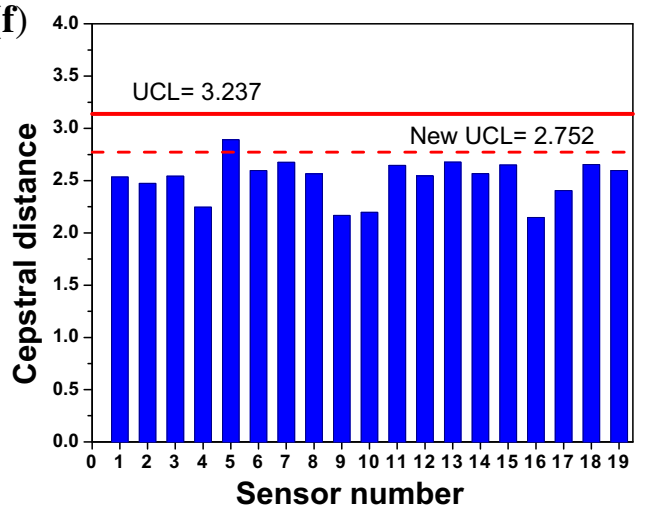

Figure 6. Damage indices of a current data of single damage scenario. (a) First subset with SNR 40, (b) fifth subset with SNR 40, (c) first subset with SNR 50, (d) fifth subset with SNR 50, (e) first subset with SNR 60 and (f) fifth subset with SNR 60.

UCL calculated in this example is not able to indicate the outlier properly. This could be due to the reason that the novelty indices are calculated from the damage indices which are obtained from the healthy subsets without any normalization process. This increases the magnitude of the damage indices and subsequently increases the magnitude of the control limits used to detect the damage. Therefore, when a new current subset is employed for diagnosis, the damage index even though shows a rough notion of the damage location does not become an outlier.

A similar trend is seen in the current data with multiple damage scenarios where the stiffness of element 3 and 12 are reduced by $22 \%$. The damage indices along with the UCL (indicated using a solid line) are shown in figure 7 for the multiple damage scenario with SNR 40. From the figure, it is clear that the novelty index calculated from the subsets without normalization is found to be unrealistic and ineffective to indicate the location of damage.

In view of this, we propose to calculate the novelty indices by data normalization of healthy subsets. Each healthy subset is matched properly with the other healthy subsets by using the correlation coefficients of their AR coefficients. These matched subsets are used in the evaluation of damage indices and further in the calculation of control limits as in Eq. (13). These new novelty indices are expected to give a better indication of the location of damage as the control limits are established by handling the variability among the different subsets. The new UCL 

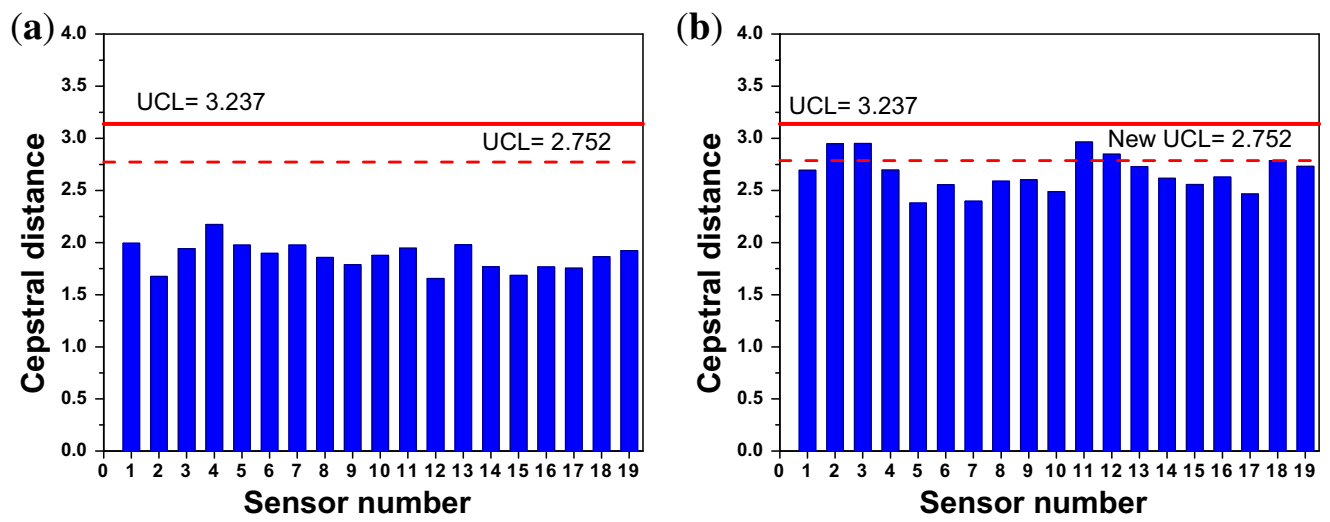

Figure 7. Damage indices of a current data corresponding to multiple damage scenario with SNR 40: (a) First subset and (b) Fifth subset.

obtained for this example using the normalization of healthy subsets is found to be 2.752 and it is plotted on figure 6 using the dashed line.

By using the new UCL, it can be seen that both the presence and the location of damage are indicated for the fifth current subsets with SNR 40, 50 and 60. From figure 6, it is clear that the novelty index calculated from the normalized healthy subsets establishes the correct control limits and are effective in damage detection.

Similarly, the multiple damage scenario is tested and the damage index is found to be indicating the location of damage exactly through the new control limits calculated from the healthy subsets matched with the other healthy subsets. The new UCL for multiple damage scenario with SNR 40 is shown with a dashed line in figure 7.

From this numerical example, it can be concluded that

- The damage index, based on cepstral distance measure, calculated from the ARMAX model, is found to be a good indicator for damage identification. However, for civil engineering problems which are subjected to environmental and operational variability, apart from the measurement noise, it may not be effective, unless it is combined with novelty analysis.

- It is also shown that the control limits need to be established from the healthy subsets combining with the matching procedure in order to effectively identify the time instant of damage and also spatial location.

- Novelty indices are much useful for the damage diagnosis, where the variability due to the environment, operational conditions and measurement noise are present in the measured data.

\subsection{LANL 8-DOF system}

The experimental datasets of eight degree-of-freedom system, designed and constructed by Los Alamos National Laboratory [19] are used in the present work to study the

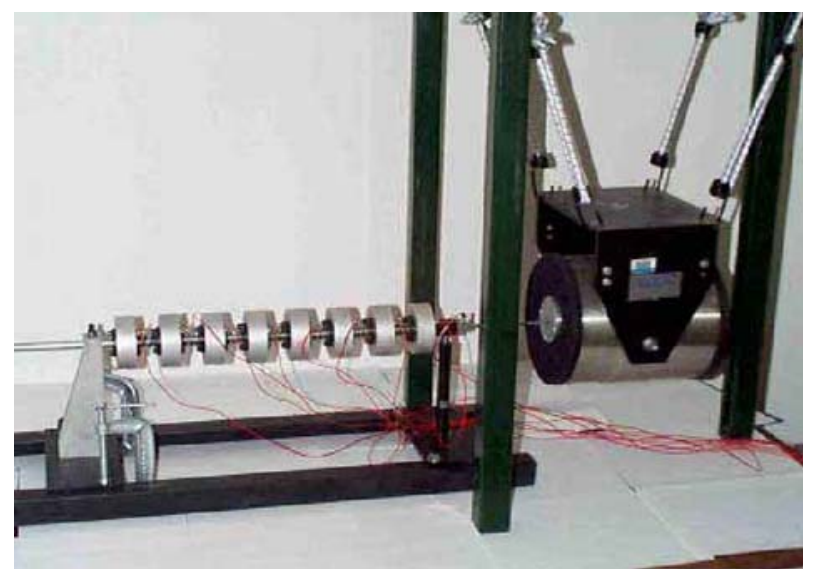

Figure 8. Excitation of 8-DOF system with electro-dynamic shaker.

effectiveness of the proposed damage identification technique based on ARMAX model. The system consists of eight translational masses which are aluminium discs of $25.4 \mathrm{~mm}$ thick and $76.2 \mathrm{~mm}$ in diameter connected with springs as shown in figure 8 . Linear damage is simulated by replacing an original spring with another with a lesser spring constant. The following parameters are used in the acceleration time history responses provided in the website.

Maximum frequency: $200 \mathrm{~Hz}$

Number of frequency lines: 1600

Record length: $8 \mathrm{~s}$

Sample rate: $500 \mathrm{~Hz}$

Number of data points: 4096.

4.2a Formation of baseline and current data: Two scenarios based on the type of excitation (random and impulse) named as Feb198 and Apr23pr as shown in table 1 are considered for the present investigations. All the datasets of Feb198 and Apr23pr corresponding to the undamaged (with original spring stiffnesses) state of the system are combined together, in order to simulate 
Table 1. Details of damage scenarios and their data files obtained from 8-DOF system.

\begin{tabular}{|c|c|c|c|c|}
\hline S. no & Damage scenario & Damage condition & Test data file & Type of excitation \\
\hline \multirow[t]{8}{*}{1} & \multirow[t]{8}{*}{ Feb 198} & \multirow[t]{4}{*}{ Undamaged } & Feb 198u1 & Random, $6 \mathrm{v}$ \\
\hline & & & Feb 198u2 & Random, $5 \mathrm{v}$ \\
\hline & & & Feb 198u3 & Random, $4 \mathrm{v}$ \\
\hline & & & Feb 198 u 4 & Random, $3 \mathrm{v}$ \\
\hline & & \multirow[t]{4}{*}{ Damaged (14\% damage at Location 5) } & Feb 198d5 & Random, 3v \\
\hline & & & Feb $198 \mathrm{~d} 8$ & Random, 4v \\
\hline & & & Feb 198d9 & Random, 5v \\
\hline & & & Feb $198 d 10$ & Random, 6v \\
\hline \multirow{9}{*}{2} & \multirow[t]{9}{*}{ Apr23pr } & \multirow[t]{5}{*}{ Undamaged } & Apr23pr1 & \multirow[t]{5}{*}{ Impulse } \\
\hline & & & Apr23pr2 & \\
\hline & & & Apr23pr3 & \\
\hline & & & Apr23pr4 & \\
\hline & & & Apr23pr5 & \\
\hline & & \multirow[t]{4}{*}{ Damaged (14\% damage at Location 5) } & Apr23pr7 & \multirow[t]{4}{*}{ Impulse } \\
\hline & & & Apr23pr8 & \\
\hline & & & Apr23pr9 & \\
\hline & & & Apr23pr10 & \\
\hline
\end{tabular}
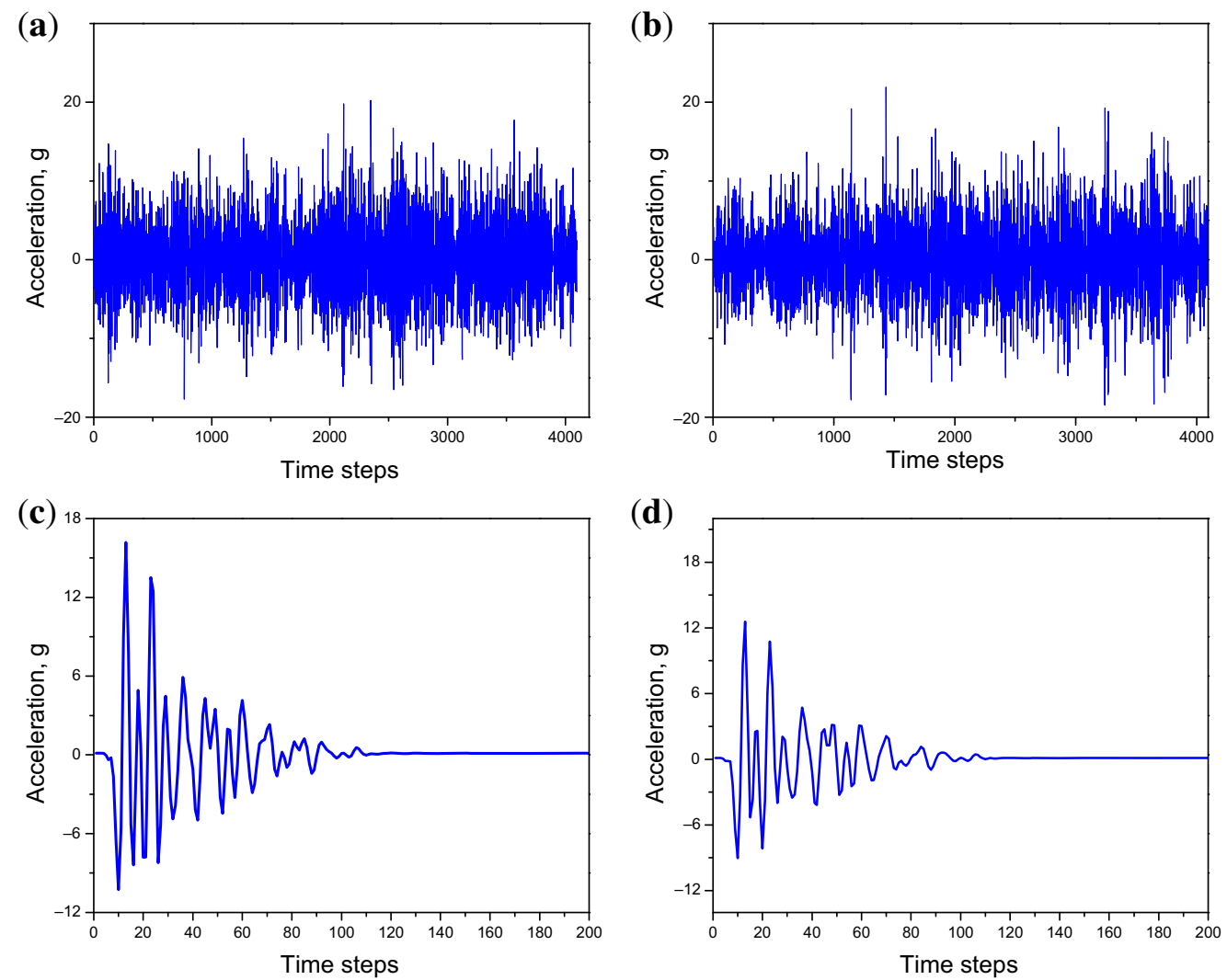

Figure 9. Typical acceleration data of channel 5 of 8-DOF system: (a) FEB198 scenario-healthy, (b) FEB198 scenario-current data and (c) APR23 scenario-healthy and (d) APR23 scenario-current data.

different operational conditions. This makes the baseline data pool with nine datasets of 4096 time points each. The baseline data pool is then subdivided into 56 subsets of 512 data points each (i.e. every subset data has a time duration of $1 \mathrm{~s}$ ). These healthy baseline subsets are used to calculate the upper control limits on the damage indices, as described in the previous example, using Eq. (13). The control limits are then used to compare the damage indices of any new current data subset to decide upon the state of the structure. Every dataset of Feb198 and Apr23rpr, recorded from the system after the simulation of damage (14\% stiffness reduction at location 5) are divided 

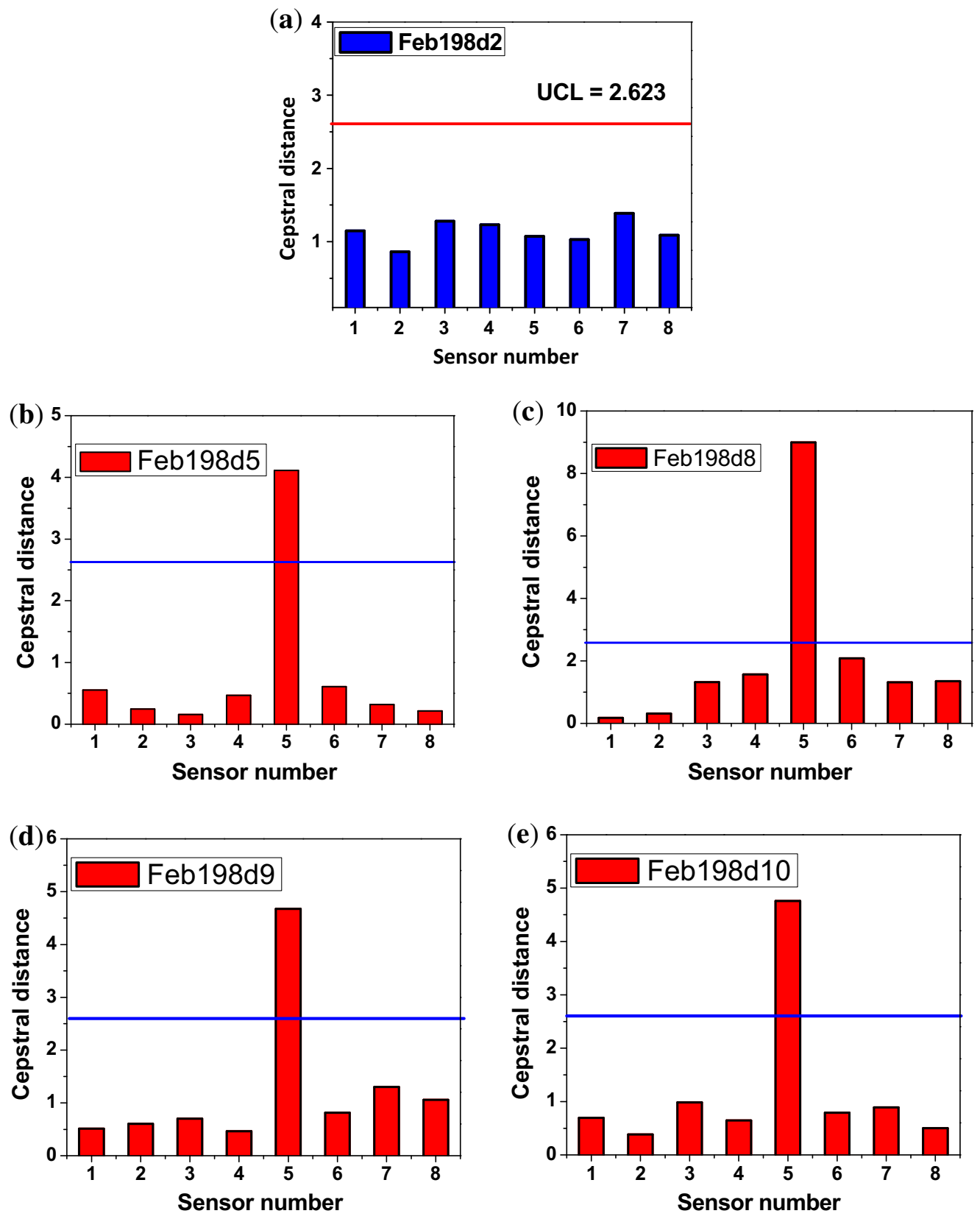

Figure 10. Cepstral distances of ARMAX models corresponding to Feb198 damage scenarios of 8-DOF system.

into eight subsets of 512 data points each and are combined with any two healthy baseline subsets to form current data subsets. Here, by forming the current data as outlined above, a situation is simulated where initially the structure is healthy and after $2 \mathrm{~s}$ the damage gets initiated. Typical acceleration time history data of a healthy and a current dataset for Feb198 and Apr 23pr scenarios are shown in figure 9.

4.2b Investigations of Feb198 scenario: Every current data subset is fitted to the ARMAX model. Using the AR coefficients thus obtained, they are subjected to the normalization process described in section 3.2. Then the damage indices are calculated from the zeros and poles of the matched data subsets as described in section 3.3. The damage index, thus obtained for the first current subset is shown in figure 10(a). A close look at figure 10(a) indicates that the cepstral distances computed for all the nodes are found to be well within the UCL of 2.623 , calculated as discussed in the previous example. From this, it can be concluded that the first current data subset is obtained when the system is healthy. Similarly, the second data subset also showed a similar trend, 

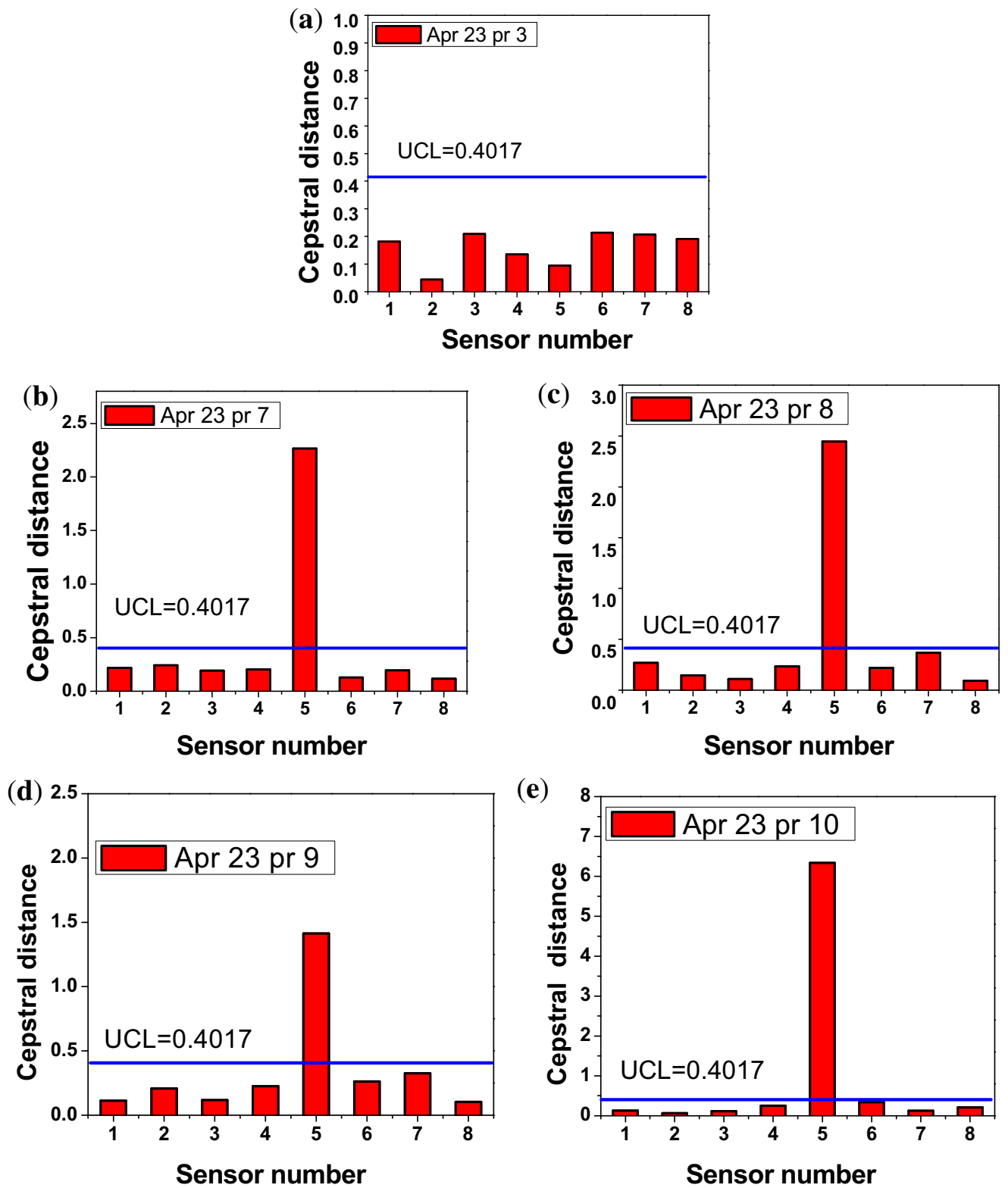

Figure 11. Cepstral distances of ARMAX models corresponding to Apr23pr damage scenarios of 8-DOF system.

where the cepstral distances lie within the UCL. When the third subset is tested, the magnitudes of the cepstral distances peak up beyond the UCL, near sensor location 5, for all the damage test cases of Feb198. This is clearly shown in figure 10(b)-10(e), for all the test datasets considered, indicating the precise spatial location of damage as element no 5.

4.2c Investigations of Apr23pr scenario: Similar to the procedures explained in the earlier scenario (Feb198), every current dataset of Apr23pr scenario is subjected to damage detection using the proposed technique discussed in sections 3.2 and 3.3. The results of the studies using different datasets are shown in figure 11 . It can be easily verified from the plots given in figure 11(a) that the cepstral distances computed are found to be well within the UCL for the first current subset. From this, it is clear that the first current data subset is obtained when the system is healthy. A similar trend is found for any healthy subset. When the third data subset is diagnosed, damage indices at sensor location five show high magnitudes clearly indicating the spatial location of damage. This is found to be true for all the test cases used for investigation. The cepstral distances of ARMAX models of different test datasets of APR23pr scenario are shown in figure 11(b)-11(e). From the figures, it can be seen that the technique is capable of detecting and locating the damage in the presence of operational variability. 


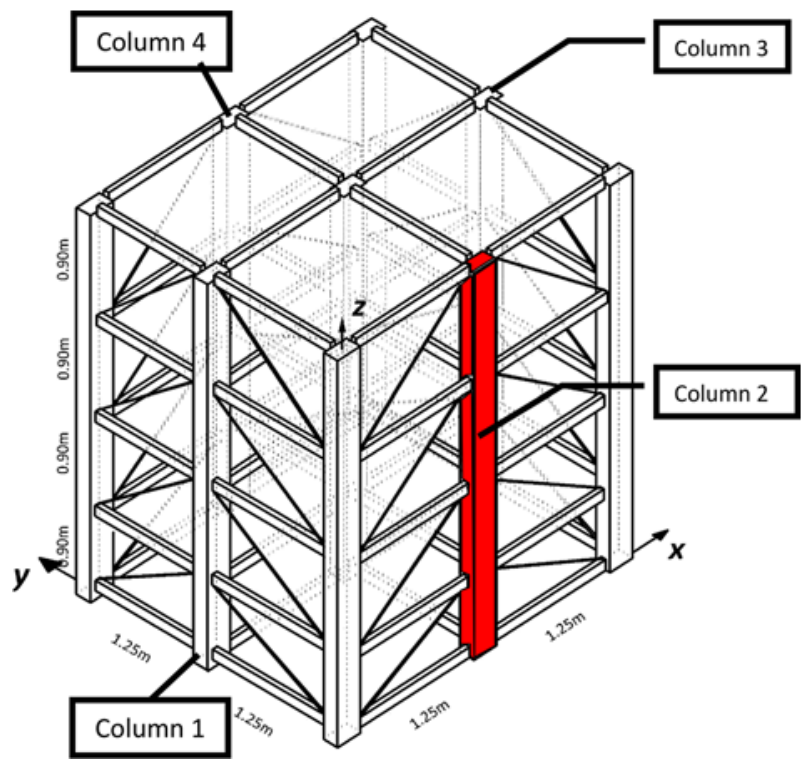

Figure 12. Analytical model of IASC-ASCE steel frame.

Table 2. Details of damage patterns adopted for 12-DOF system of ASCE benchmark structure.

\begin{tabular}{lc}
\hline $\begin{array}{l}\text { Damage } \\
\text { pattern }\end{array}$ & Description of damage \\
\hline 1 & All braces of 1st storey are broken \\
2 & All braces of 1st and 3rd storey are broken \\
3 & 1 brace on 1side of 1st storey is broken \\
4 & 1 brace on 1 side of 1st and 3rd storey are broken \\
5 & Pattern $4+$ unscrew the left end of element 18 \\
6 & Area of 1 brace on 1 side of 1st storey is reduced \\
& to $2 / 3$ \\
\hline
\end{tabular}

\subsection{IASC-ASCE Phase I SHM benchmark problem [20]}

IASC-ASCE Phase I SHM benchmark structure is a fourstorey, two-bay by two-bay steel-frame quarter-scale model structure fabricated in the Earthquake Engineering Research Laboratory at the University Of British Columbia (UBC). The plan dimensions of the scaled down model are $2.5 \mathrm{~m} \times 2.5 \mathrm{~m}$ and the height is $3.6 \mathrm{~m}$. Further details on this model can be found in Johnson et al [20].

A finite element model (12-DOF shear building model) based on this structure developed by ASCE task group is used to generate the simulated response data for this study. The analytical model of the structure is shown in figure 12 . It may be noted that $\mathrm{x}$-direction (i.e., bending about the $\mathrm{y}$ axis) is the strong direction due to the orientation of the columns. The compass directions associated with the axes are South for the positive $\mathrm{y}$ (weak) direction, and West for the positive $\mathrm{x}$ (strong) direction.

Twelve-DOF shear building finite element model is used to generate the simulated data for the SHM benchmark problem for healthy as well as damaged state. In this model, the floors (i.e., floor beams and floor slabs) move as rigid bodies, with translation in the $x$ and $y$ directions and rotation $\theta$ about the center column. Thus, there are 3 DOF per floor. The columns are modeled as linear elastic EulerBernoulli beams, and the braces as axial bars. The acceleration time history data are obtained from 12 DOFs from four floors. However, in the present investigations, we have considered only the acceleration data in the X-direction in each floor on column 2 , indicated in figure 12 , for damage diagnosis. The numerical simulations are carried out for 10 s with sampling rate as $2000 \mathrm{~Hz}$. The noise level in the simulations is considered as $10 \%$ of the largest RMS of the acceleration responses (typically one of the roof accelerations). (a)

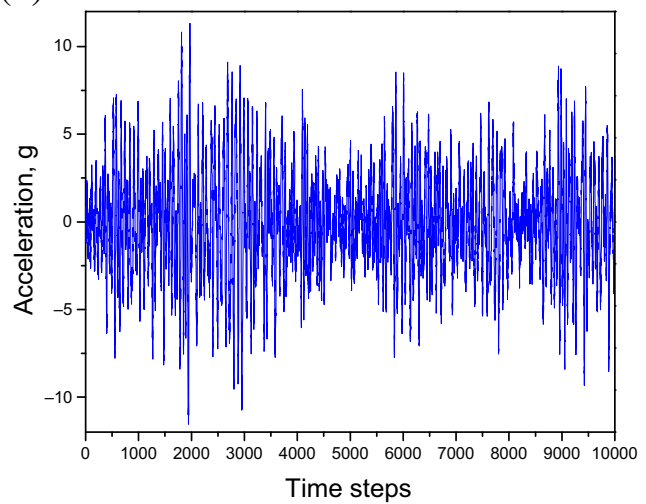

(b)

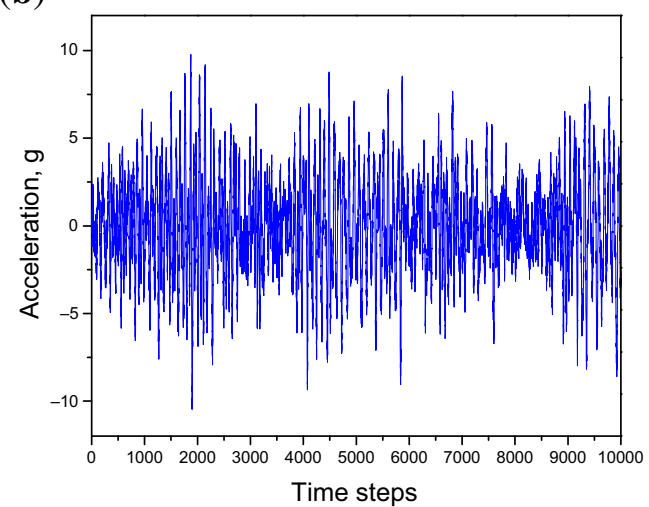

Figure 13. Typical first floor accelerations of column 2 in X-direction: (a) healthy data and (b) current data of damage pattern 1. 

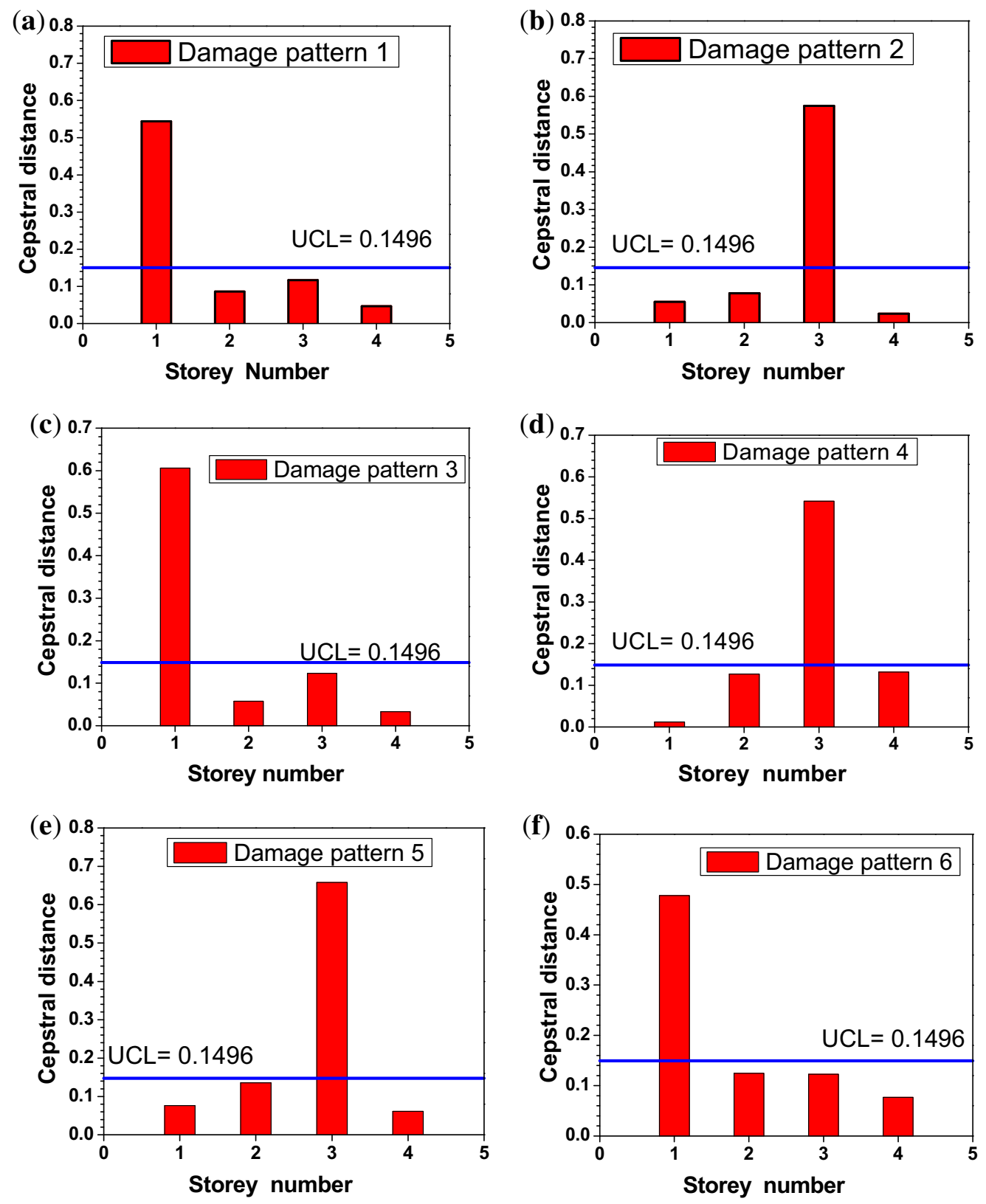

Figure 14. Cepstral distances of ARMAX models corresponding to damage patterns 1-6 of 12- DOF system of ASCE benchmark structure.

Six damage patterns shown in table 2 are investigated in the present work to demonstrate the effectiveness of the proposed damage detection algorithm in detecting and locating both single and multiple damages. These damage patterns vary from a very simple, extreme damage (Pattern1 ), that most of the methods should be able to detect, to more complex damage scenarios. The following points on the damage patterns given table 2 are appropriate to note for the shear building model.

1. In damage pattern-1, even though all braces of the $1 \mathrm{st}$ storey are broken, it can be considered as a single damage case as the damage is to be assessed only from one DOF in the first floor in the shear building model.

2. Damage patterns 1, 3 and 6 are treated as single damage scenarios and the other patterns are used to simulate multiple damage scenarios.

3. Damage pattern-4 is no different than pattern-5 for the 12-DOF model.

4. Damage patterns 2, 4 and 5 are considered as the subsequences of damage patterns 1,3 and 6 respectively.

The time history data is collected for $20 \mathrm{~s}$ and the total number of samples collected for healthy structure comes to 40,000. Typical acceleration time history data of sensor at 
Table 3. Performances of damage identification of IASC-ASCE benchmark structure using different techniques.

\begin{tabular}{lcccccccc}
\hline & \multicolumn{7}{c}{ Damage identification methods as per section 4.3a } \\
\cline { 2 - 7 } Damage pattern & 1 & 2 & 3 & 4 & 5 & 6 & 7 & Present method \\
\hline 1 & $\mathrm{Y}$ & $\mathrm{Y}$ & $\mathrm{Y}$ & $\mathrm{N}$ & $\mathrm{N}$ & $\mathrm{Y}$ & $\mathrm{Y}$ & $\mathrm{Y}$ \\
2 & $\mathrm{Y}$ & $\mathrm{Y}$ & $\mathrm{Y}$ & $\mathrm{N}$ & $\mathrm{N}$ & $\mathrm{Y}$ & $\mathrm{Y}$ & $\mathrm{Y}$ \\
3 & $\mathrm{Y}$ & $\mathrm{Y}$ & $\mathrm{Y}$ & $\mathrm{Y}$ & $\mathrm{N}$ & $\mathrm{N}$ & $\mathrm{Y}$ & $\mathrm{Y}$ \\
4 & $\mathrm{Y}$ & $\mathrm{Y}$ & $\mathrm{Y}$ & $\mathrm{N}$ & $\mathrm{N}$ & $\mathrm{N}$ & $\mathrm{Y}$ & $\mathrm{N}$ \\
5 & $\mathrm{~N}$ & $\mathrm{~N}$ & $\mathrm{~N}$ & $\mathrm{~N}$ & $\mathrm{Y}$ & $\mathrm{N}$ & $\mathrm{Y}$ \\
6 & $\mathrm{~N}$ & $\mathrm{~N}$ & $\mathrm{~N}$ & $\mathrm{~N}$ & $\mathrm{Y}$ & $\mathrm{N}$ & $\mathrm{N}$ \\
\hline
\end{tabular}

column number 2 of the first floor, before and after the damage pattern 1 is simulated are shown in figure 13 for a length of 1000 data points.

The data is divided into 80 subsets with 500 samples in each subset. These healthy subsets are used in the calculation of UCL using the procedure described in the first example. Now, to test any current data, it is assumed that the structure is healthy initially for $2 \mathrm{~s}$ and then the damage as per the patterns given in table 2 are simulated. The time history data with the simulated damage is compiled for carrying out the investigations on the proposed damage detection technique. The cepstral distances of two matched ARMAX models obtained for different test data subsets are presented in figure 14. As mentioned earlier, the damage patterns 2, 4 and 5 are considered as the subsequence of damage patterns 1,3 and 4 respectively. In order to accomplish this, once the damage pattern- 1 is located the damaged datasets corresponding to the damage pattern- 1 is added in the healthy data pool to locate the 2 nd (subsequent) damage in pattern-2. This can be viewed in such a way that the damage pattern- 2 is considered as the subsequence of damage pattern- 1 and can be argued that there always exists minimum time lag between the incipience of any subsequent spatial damages. Similar sort of procedure is adopted for damage pattern-4 and 5. Figure 14 clearly shows the spatial location of all the damage patterns robustly.

4.3a Comparison with other detection methods [21]: The damage Identification of IASC-ASCE benchmark structure, through six different damage patterns, defined by SHM Task Group, has been widely investigated by the various SHM methods such as (1) time series analysis method [22], (2) flexibility based approach [23], (3) wavelet method [24], (4) wavelet packet transform [25], (5) Bayesian model updating [26], (6) Hilbert-Huang Transform-based method [27], and (7) adaptive RLS filters [28]. The identification performances of these methods are listed in table 3 . In the table, "Y" denotes that the damage pattern can be detected using the corresponding method and " $\mathrm{N}$ " denotes that the damage pattern is either not considered for testing or cannot be identified using the particular method. From table 3, it can be seen that the proposed technique can identify all the six damage patterns, ranging from severe to mild damage scenario.

\section{Conclusion}

In this paper, a damage localization method based on ARMAX model and the Cepstral distances as damage indicator is presented. The proposed method is shown to be effective in detecting the spatial location of damage, by utilizing an example of a simply supported beam with variability due to temperature and operational loads along with the measurement noise. The standard benchmark data from 8-DOF spring-mass system of EI-LANL and the simulated FEM 12-DOF shear building model of IASCASCE steel frame are used for validation of the proposed technique. All the acceleration signals are made uncorrelated before using the damage indicator to improve the robustness of the technique. A normalization process is proposed using the AR coefficients obtained from the fitting of ARMAX models to the datasets. In addition to this, novelty index is employed to arrive at the control limits in order to clearly differentiate the damage from the effects of other variability. The results of the studies show that the proposed technique is effective in identifying the time instant and the spatial location of the damage effectively.

\section{Acknowledgement}

This paper is being published with the permission of the director, CSIR-Structural Engineering Research Centre, Taramani, Chennai.

\section{References}

[1] Lynch J P, Sundararajan A, Law K H, Kiremidjian A S and Carryer E 2004 Embedding damage detection algorithms in a wireless sensing unit for attainment of operational power efficiency. Smart Mater. Struct. 13(4): 800-810

[2] Straser E G and Kiremidjian A S 1998 Modular wireless damage monitoring system for structures, Report No. 128, John A. Blume Earthquake Engineering Center, Department of Civil and Environmental Engineering, Stanford University, Stanford, CA

[3] Doebling S W, Farrar C R, Prime M B and Shevitz D W 1996 Damage identification and health monitoring of structural and mechanical systems from changes in their vibration 
characteristics: A literature review, Los Alamos National Laboratory Report LA-13070-MS, Los Alamos National Laboratory, Los Alamos, NM 87545

[4] Sohn H, Farrar C R, Hunter H F and Worden K 2001 Applying the LANL statistical pattern recognition paradigm for structural health monitoring to data from a surface-effect fast patrol boat, Los Alamos National Laboratory Report LA13761-MS, Los Alamos National Laboratory, Los Alamos, NM 87545

[5] Sohn H and Farrar C R 2001 Damage diagnosis using time series analysis of vibration signals. Smart Mater. Struct. 10: 446-451

[6] Fassois S D and Sakellariou J S 2007 Time series methods for fault detection and identification in vibrating structures. Roy. Soc. Philos. Trans.: Math., Phys. Eng. Sci. 365: 411-448

[7] Ljung L 1999 System identification: Theory for the user. 2nd edn, Prentice Hall

[8] Carden E P and Brownjohn J M 2008 Arma modelled time series classification for structural health monitoring of civil infrastructure. Mech. Syst. Signal Process. 22(2): 295-314

[9] Zhang Q W 2007 Statistical damage identification for bridges using ambient vibration data. Comput. Struct. 85: 476-485

[10] Bodeux J B and Golinval J C 2001 Application of ARMAV models to the identification and damage detection of mechanical and civil engineering structures. Smart Mater. Struct. 10: 479-489

[11] Zheng $\mathrm{H}$ and Mita A 2007 Two stage damage diagnosis based on the distance between arma models and prewhitening filters. Smart Mater. Struct. 16: 1829-1836

[12] Sakellariou J S and Fassois S D 2006 Stochastic output error vibration based damage detection and assessment in structures under earthquake excitation. J. Sound Vib. 297: 1048-1067

[13] Fung Eric H K, Wong Y K, Ho H F and Mignolet Marc P 2003 Modelling and prediction of machining errors using ARMAX and NARMAX structures. Appl. Math. Modell. 27: 611-627

[14] Zhenhua Xing Y Z and Akira Mita 2012 A substructure approach to local damage detection of shear structure. Struct. Control Health Monit. 19(2): 309-318

[15] Ay Ali M and Wang Ying 2014 Structural damage identification based on self-fitting ARMAX model and multi-sensor data fusion. Struct. Health Monit. 13(4): 445-460
[16] Oppenheim A V and Schafer R W 1975 Digital signal processing. Prentice-Hall, London, UK

[17] Luke Dosiek and John W Pierre 2013 Estimating electromechanical modes and mode shapes using the multichannel ARMAX model. IEEE Trans. Power Syst. 28(2): 1950-1959

[18] Martin R J 2000 A metric for ARMA processes. IEEE Trans. Signal Process. 48: 1164-1170

[19] http://institute.lanl.gov/ei/software-and-data

[20] Johnson E A, Lam H F, Katafygiotis L S and Beck J L 2004 Phase I IASC-ASCE structural health monitoring benchmark problem using simulated data. J. Eng. Mech.130: 3-15. DOI: 10.1061/(ASCE)0733-9399(2004)130:1(3)

[21] Futao Zhu and Yunjie Wu 2014 A rapid structural damage detection method using integrated ANFIS and interval modeling technique. Appl. Soft Comput. 25: 473-484

[22] Nair K K, Kiremidjian A S and Law A S 2006 Time seriesbased damage detection and localization algorithm with application to the ASCE benchmark structure. J. Sound Vib. 291: 349-368

[23] Bernal D and Gunes B 2004 Flexibility based approach for damage characterization: benchmark application. J. Eng. Mech.-ASCE 130: 61-70

[24] Hera A and Hou Z K 2004 Application of wavelet approach for ASCE structural health monitoring benchmark studies. $J$. Eng. Mech.-ASCE 130: 96-104

[25] Ding Y, Li A and Liu T 2008 A study on the WPT-based structural damage alarming of the ASCE benchmark experiments. Adv. Struct. Eng. 11: 121-127

[26] Ching J 2004 New Bayesian model updating algorithm applied to a structural health monitoring benchmark. Struct. Health Monit. 3: 313-332

[27] Lin S, Yang J N and Zhou L 2005 Damage identification of a benchmark building for structural health monitoring. Smart Mater. Struct. 14: S162-S169

[28] Chase J G, Begoc V and Barroso L R 2005 Efficient structural health monitoring for a benchmark structure using adaptive RLS filters. Comp. Struct. 83: 639-647

[29] Yan A M, Kerschen G, De Boe P and Golinval J C 2005 Structural damage diagnosis under varying environmental conditions-Part I: A linear analysis. Mech. Syst. Signal Process. 19: 847-864 